\title{
THE CHLORINE NEEDS OF COCONUTS
}

by

\author{
S.S. Magat and R.Z. Margate ${ }^{1}$
}

\begin{abstract}
A study designed to determine the nutritional needs of coconut from nursery to full-bearing was conducted from 1974 to 1988 in a Cl-deficient soil (Topic Tropudalf) of Davao, Southern Philippines.

At nursery stage, only chlorine application improved the growth of the seedlings based on girth size which was highly correlated with increased leaf $\mathrm{Cl}$. During the first five years in the field, $\mathrm{K}$ and $\mathrm{Cl}$ significantly influenced the growth of the palms which were positively correlated to ail growth parameters, i.e. girth, number of leaflets, living fronds and leaf production. During the fifth year, Cl-fertilization resulted in $115 \%$ more flowering palms than the unfertilized ones. From then on, $\mathrm{Cl}$ application consistently increased nut production, copra weight per nut and copra yield per tree which was correlated with leaf $\mathrm{Cl}$. However, the positive effects of other nutrients like $\mathrm{N}, \mathrm{K}$ and $\mathrm{Mg}$ on yield as well as interaction effects were inconsistent over the years.

The nutritional needs of palms for $\mathrm{Cl}$ at the nursery stage is $30 \mathrm{~g} \mathrm{Cl}$ per seedling. Under field condition, the requirement increases with age from $40 \mathrm{~g}$ to $1,000 \mathrm{~g}$ per palm leveling off at fifth year from planting.
\end{abstract}

\section{INTRODUCTION}

Several studies have been conducted on coconut fertilization in the country and elsewhere. Nevertheless the nutritional balance particularly in $\mathrm{N}, \mathrm{K}, \mathrm{Mg}$ and $\mathrm{Cl}$ for growth and production from nursery to full-bearing stages has not been established, particularly in inland coconuts.

The need for $\mathrm{C} 1$ in the vegetative and reproductive growth of coconut palms was initially demonstrated at the Davao Research Center (Mendoza and Prudente, 1972). They later confirmed that palms fertilized with $\mathrm{KCl}$ together with either $\mathrm{N}$ or NP produced significantly more nuts with thicker meat and more copra per palm than the unfertilized ones (Prudente and Mendoza, 1976). Related studies revealed that most inland coconuts apparently respond to $\mathrm{N}$ and $\mathrm{Cl}$ (Magat et al, 1975) and that the positive effect of $\mathrm{Cl}$ is manifested more in terms of copra weight per nut (Magat and Prudente, 1975; and Margate et al, 1978).

Studies on the effect of $\mathrm{Cl}$ on coconut seedlings indicated that both $\mathrm{KCl}$ and $\mathrm{NaCl}$ positively influence the growth of seedlings and increase their resistance to leaf spot disease (Abad et al 1978; Magat et al, 1977).

During the period 1974 to 1988 an experiment was conducted at the Davao Research Center to assess the $\mathrm{Cl}$ nutritional needs of coconuts. Specifically, under field conditions it was designed to determine the coconut's critical and optimum level for $\mathrm{Cl}$ and the need for $\mathrm{N}, \mathrm{K}$ and $\mathrm{Mg}$ using a Cl-deficient soil.

\footnotetext{
${ }^{1}$ Senior Research Staff, Agricultural Research Management Department, Philippine Coconut Authority, Diliman, Quezon City, and Davao Research Center, Philippine Coconut Authority, Davao City, Philippines, respectively.
} 


\section{MATERIALS AND METHODS}

\section{Planting Materials and growing conditions}

The planting materials used in the study belong to the tall 'Laguna' variety. The seedlings were raised in a polybag nursery $(60 \mathrm{~cm} \times 60 \mathrm{~cm}$ triangular system, N-S orientation) for seven months and were field planted in August 1974 at a spacing of $9 \mathrm{~m} \times 9 \mathrm{~m}$ in triangular pattern, following a N-S orientation.

The soil in the experimental area is classified as Tugbok day loam (Typic Tropudalf) with physical and chemical properties as shown in appendix Table 1. It has an acceptable internal and external drainage.

Rainfall is almost evenly distributed throughout the year; and sunshine, relative humidity and temperature at satisfactory levels for coconuts. For the past ten years, average rainfall and relative humidity were respectively 2, $102 \mathrm{~mm}$ (annual) and 80.5\% (monthly).

\section{Layout and treatments}

A $3^{3}$ factorial experiment in a split-plot, incomplete block design was adopted in the study. The split plots were either with or without $\mathrm{Cl}$, with 27 main plots and 54 sub-plots. The three other factors being investigated were $\mathrm{N}, \mathrm{K}$ and $\mathrm{Mg}$. There were 12 palms per elementary plot with common guard rows. The elements being studied and their sources were as follows:

1) $\left.\mathrm{N}-\left(\mathrm{NH}_{4}\right)_{2} \mathrm{SO}_{4}, \mathrm{NH}_{4} \mathrm{C}\right)$

2) $\mathrm{K}-\mathrm{KCl}$ and $\mathrm{K}_{2} \mathrm{SO}_{4}$

3) $\mathrm{Mg}-\mathrm{CaMgCO}_{3}$ (dolomite)

4) $\mathrm{Cl}-\mathrm{KCl}$ and $\mathrm{NH}_{4} \mathrm{Cl}$

In the course of the study, an unusual yellowing of the fronds was observed two years from planting. This was confirmed later to be due to $\mathrm{S}$ deficiency. To avert the situation, a blanket application of gypsum at the rate of $2 \mathrm{~kg}$ per palm per year was done starting October 1976.

The annual fertilizer rates (Table 1) were applied in split at equal doses during the months of February and August.

\section{Parameters}

Data on growth characters such as girth, leaf emission and count, number of leaflets (rank 3), trunk height, flowering precocity and yield were variedly taken, depending on the age of the palms. Leaf samples were collected and analyzed periodically for concentrations of $\mathrm{N}, \mathrm{P}, \mathrm{K}, \mathrm{Ca}, \mathrm{Mg}, \mathrm{Na}, \mathrm{Cl}$, $\mathrm{S}$ and $\mathrm{B}$, at the PCA's Tissue Analysis Laboratory, HQ, Diliman, Quezon City.

\section{RESULTS AND DISCUSSION}

\section{Effect of nutrients on growth of seedlings (nursery stage)}

Table 2 shows a clear manifestation of the response of the seedlings to $\mathrm{Cl}$ application in terms of girth circumference compared with the control in ail stages of observations. Nitrogen and $\mathrm{Mg}$ did not have any influence on the growth of the seedlings. But $\mathrm{K}$ at a lower level, gave some positive effect. The findings reveal that the application of both $\mathrm{K}$ and $\mathrm{Cl}$ is beneficial to coconut seedlings. This positive response to $\mathrm{Cl}$ strongly indicates the deficiency of the Tugbok soil in this element, more so that the site of the study is far ( $8 \mathrm{~km}$ away) from the sea. Results were consistent with earlier studies of Magat et. al. (1977): Maravilla et al (1978) and Oguis et al (1979). 


\section{Effect of nutrient on young field-planted palms}

As early as six months from field planting up to the second year, practically all palms exhibited yellowish-orange leaves especially on older ones. After a close investigation, it was confirmed to be due to $\mathrm{S}$ deficiency more particularly in palms applied with $\mathrm{Cl}$ due to $\mathrm{Cl}-\mathrm{S}$ antagonism. So, starting October 1976, a blanket application of gypsum $\left(\mathrm{CaSO}_{4} \cdot \mathrm{NH}_{2} \mathrm{O}\right)$ as a source of $\mathrm{S}$ was made. Henceforth, the subplots were changed from $+\mathrm{Cl}$ and $+\mathrm{S}$ to $+\mathrm{Cl}$ and $-\mathrm{Cl}$.

Six months after application of fertilizers, treatments $\mathrm{KI}, \mathrm{Mgl}$ and $\mathrm{Cl}$ significantly increased the girth of the palms (Table 3). Similarly in succeeding years the palms showed significant response to $\mathrm{K}, \mathrm{Mg}$ and $\mathrm{Cl}$ application. The effects were more manifested in the girth and number of leaflets of the palms. In older palms, both $\mathrm{K}$ and $\mathrm{Cl}$ treated plots consistently produced palms with more living fronds, higher leaf production, more accumulated leaf production and taller palms. The lack of positive response to $\mathrm{N}$ application is very likely due to the benefits obtained from the well-established cover-crops which could have maintained a high soil level of $\mathrm{N}$.

Details of the results in the nursery and in young field planted palms were earlier reported (Magat and Oguis, 1979).

\section{Effect of nutrient on yield}

a. Nut per palm - For the last five years (1984-88) of production (Table 4) it was only $\mathrm{Cl}(1$ $\mathrm{kg} / \mathrm{palm}$ ) that gave consistent trend of response over the minus $\mathrm{Cl}$ treatment. Other elements like $\mathrm{N}$, $\mathrm{K}$, and $\mathrm{Mg}$ did not produce any significant effect on nut production in ail the years. The nut production of the $\mathrm{Cl}$-fertilized palms ranged on the average at 47-85 nuts per year while the minus $\mathrm{Cl}$ palms with 26-63 nuts, corresponding to a 50\% increase in nuts due to Cl-fertilization. The results generally indicate that $\mathrm{Cl}$ is the major limiting factor in the area of study.

b. Copra weight per nut - Table 5, shows the effect of the different treatments on copra weight per nut. Again, in ail the years of production $\mathrm{Cl}$ significantly improved the weight of copra per nut over the minus $\mathrm{Cl}$ treatment. Over the years, average copra per nut of $231 \mathrm{~g}$ and $300 \mathrm{~g}$ of unfertilized and $\mathrm{Cl}$-fertilized palms, respectively were noted. This indicates a $30 \%$ increase in copra weight per nut with $\mathrm{Cl}$ fertilization. Magnesium did not produce any effect at ail while K1 $(425 \mathrm{~g} \mathrm{~K})$ and K2 (850 g K) significantly increased the weight of copra per nut except in 1985 and 1987. However, compared to $\mathrm{Cl}$ application (30\% increase), $\mathrm{K}$ application increased copra weight per nut by only $7 \%$ over unfertilized palms. Nitrogen at $\mathrm{N} 2(140 \mathrm{~g} \mathrm{~N})$ produced significant improvement especially for the last four years of production, while $\mathrm{N}$, had been very inconsistent.

c. Copra weight per palm - Just like nut yield. the response was more clue to $\mathrm{Cl}(1 \mathrm{~kg} \mathrm{Cl})$ fertilization in ail years (last 5 years) (Table 6). With Cl-fertilization, the average copra yield was 19 $\mathrm{kg} /$ tree or a $71 \%$ increase over the minus CI (11.2 kg copra per tree/year). Potassium application (425 $\mathrm{g} \mathrm{K}$ and $850 \mathrm{~g} \mathrm{~K})$ as well as $\mathrm{N}$ applications (140 $\mathrm{g} \mathrm{N}$ and $280 \mathrm{~g} \mathrm{~N})$ significantly affected copra yield in some years only. As in copra weight per nut, significant increases in annual copra yield per tree were inconsistent and lower from $\mathrm{K}$ and $\mathrm{N}$ application than due to $\mathrm{Cl}$-fertilization. Magnesium did not show any significant effect at ail in all the years of production. 
Table l. Fertilizer treatment applied in the nursery period and in the field (annually)

\begin{tabular}{|c|c|c|c|c|c|}
\hline \multirow[b]{2}{*}{ AGE/YEAR } & \multicolumn{5}{|c|}{ FERTILIZER (g palm)* } \\
\hline & $\begin{array}{l}\mathrm{NH}_{4} \mathrm{Cl} \\
(\mathrm{AC})\end{array}$ & $\begin{array}{c}\left(\mathrm{NH}_{4}\right)_{2} \mathrm{SO}_{4} \\
(\mathrm{AS})\end{array}$ & $\begin{array}{l}\mathrm{KCl} \\
(\mathrm{KC}) \\
\end{array}$ & $\begin{array}{c}\mathrm{K}_{2} \mathrm{SO}_{4} \\
(\mathrm{KS})\end{array}$ & $\begin{array}{c}\mathrm{CaMgCO}_{3} \\
(\mathrm{Col}) \\
\end{array}$ \\
\hline $\begin{array}{l}\text { Nursery } \\
2 \text { mos. } \\
\text { (1974) }\end{array}$ & $\begin{array}{l}\text { N0 (0) } \\
\text { N1 (5) } \\
\text { N2 (15) }\end{array}$ & $\begin{array}{l}\text { N0 (0) } \\
\text { N1 (9) } \\
\text { N2 (18) }\end{array}$ & $\begin{array}{l}\text { K0 (0) } \\
\text { K1 (10) } \\
\text { K2 }(20\end{array}$ & $\begin{array}{l}\text { K0 (0) } \\
\text { K1 (12) } \\
\text { K2 (25) }\end{array}$ & $\begin{array}{l}\operatorname{Mg} 0(0) \\
\operatorname{Mg} 1(5) \\
\operatorname{Mg} 2(10)\end{array}$ \\
\hline $\begin{array}{l}5 \text { mos. } \\
(1974)\end{array}$ & $\begin{array}{l}\text { N0 (0) } \\
\text { N1 (15) } \\
\text { N2 (30) }\end{array}$ & $\begin{array}{l}\text { N0 (0) } \\
\text { N1 (18) } \\
\text { N2 (35) }\end{array}$ & $\begin{array}{l}\text { K0 (0) } \\
\text { K1 (20) } \\
\text { K2 (40) }\end{array}$ & $\begin{array}{l}\text { K0 }(0) \\
\text { K1 }(25) \\
\text { K2 }(50)\end{array}$ & $\begin{array}{l}\operatorname{Mg} 0(0) \\
\operatorname{Mg} 1(10) \\
\operatorname{Mg} 2(20)\end{array}$ \\
\hline $\begin{array}{l}\text { Field } \\
\text { at planting } \\
\text { (Aug. 1974) }\end{array}$ & $\begin{array}{l}\text { N0 (0) } \\
\text { N1 (30) } \\
\text { N2 (60) }\end{array}$ & $\begin{array}{l}\text { N0 (0) } \\
\text { N1 (35) } \\
\text { N2 (70) }\end{array}$ & $\begin{array}{l}\text { K0 (0) } \\
\text { K1 (40) } \\
\text { K2 (80) }\end{array}$ & $\begin{array}{l}\text { K0 (0) } \\
\text { K1 (50) } \\
\text { K2 (100) }\end{array}$ & $\begin{array}{l}\operatorname{Mg} 0(0) \\
\operatorname{Mg} 1(50) \\
\operatorname{Mg} 2(100)\end{array}$ \\
\hline $\begin{array}{l}\text { Year 1 } \\
(1975)\end{array}$ & $\begin{array}{l}\text { N0 (0) } \\
\text { N1 (120) } \\
\text { N2 (240) }\end{array}$ & $\begin{array}{l}\text { N0 (0) } \\
\text { N1 (140) } \\
\text { N2 (280) }\end{array}$ & $\begin{array}{l}\text { K0 (0) } \\
\text { K1 (175) } \\
\text { K2 (350) }\end{array}$ & $\begin{array}{l}\text { K0 (0) } \\
\text { K1 (200) } \\
\text { K2 (400) }\end{array}$ & $\begin{array}{l}\operatorname{Mg} 0(0) \\
\operatorname{Mg} 1(100) \\
\operatorname{Mg} 2(200)\end{array}$ \\
\hline $\begin{array}{l}\text { Year 2 } \\
(1976)\end{array}$ & $\begin{array}{l}\text { N0 (0) } \\
\text { N1 (300) } \\
\text { N2 (600) }\end{array}$ & $\begin{array}{l}\text { N0 (0) } \\
\text { N1 }(350) \\
\text { N2 }(700)\end{array}$ & $\begin{array}{l}\text { K0 (0) } \\
\text { K1 (425) } \\
\text { K2 (850) }\end{array}$ & $\begin{array}{l}\text { K0 (0) } \\
\text { K1 (500) } \\
\text { K2 }(1000)\end{array}$ & $\begin{array}{l}\operatorname{Mg} 0(0) \\
\text { Mg1 (200) } \\
\text { Mg2 (400) }\end{array}$ \\
\hline $\begin{array}{l}\text { Year } 3 \\
(1977)\end{array}$ & $\begin{array}{l}\text { N0 (0) } \\
\text { N1 (450) } \\
\text { N2 (900) }\end{array}$ & $\begin{array}{l}\text { N0 (0) } \\
\text { N1 (550) } \\
\text { N2 (1100) }\end{array}$ & $\begin{array}{l}\text { K0 (0) } \\
\text { K1 }(600) \\
\text { K2 }(1200)\end{array}$ & $\begin{array}{l}\text { K0 (0) } \\
\text { K1 (700) } \\
\text { K2 (1400) }\end{array}$ & $\begin{array}{l}\operatorname{Mg} 0(0) \\
\text { Mg1 (250) } \\
\text { Mg2 (500) }\end{array}$ \\
\hline $\begin{array}{l}\text { Year } 4 \\
(1979)\end{array}$ & $\begin{array}{l}\text { N0 (0) } \\
\text { N1 }(500) \\
\text { N2 }(1000)\end{array}$ & $\begin{array}{l}\text { N0 (0) } \\
\text { N1 }(550) \\
\text { N2 }(1100)\end{array}$ & $\begin{array}{l}\text { K0 (0) } \\
\text { K1 (700) } \\
\text { K2 }(1400)\end{array}$ & $\begin{array}{l}\text { K0 (0) } \\
\text { K1 (800) } \\
\text { K2 }(1600)\end{array}$ & $\begin{array}{l}\operatorname{Mg} 0(0) \\
\operatorname{Mg} 1(300) \\
M g 2(600)\end{array}$ \\
\hline $\begin{array}{l}\text { Year 5-14 } \\
(1980-1988)\end{array}$ & $\begin{array}{l}\text { N0 (0) } \\
\text { N1 (600) } \\
\text { N2 (1200) }\end{array}$ & $\begin{array}{l}\text { N0 (0) } \\
\text { N1 (700) } \\
\text { N2 (1400) }\end{array}$ & $\begin{array}{l}\text { K0 (0) } \\
\text { K1 (850) } \\
\text { K2 (1700) }\end{array}$ & $\begin{array}{l}\text { K0 (0) } \\
\text { K1 (1000) } \\
\text { K2 (2000) }\end{array}$ & $\begin{array}{l}\text { Mg0 (0) } \\
\text { Mg1 (400) } \\
\text { Mg2 (800) }\end{array}$ \\
\hline $\begin{array}{l}* \mathrm{AC}-15 \% \mathrm{~N} . \\
\mathrm{KS}-50 \% \mathrm{~K} 20\end{array}$ & $\begin{array}{l}55 \% \mathrm{Cl} \\
18 \% \mathrm{~S}\end{array}$ & $\begin{array}{l}\text { AS-20\% N. } \\
\text { DOL-20\% } \\
\text { Mg }\end{array}$ & $\begin{array}{l}24 \% \mathrm{~S} \\
40 \% \mathrm{Ca} 0\end{array}$ & KC-60\% K20 & $40 \% \mathrm{Cl}$ \\
\hline
\end{tabular}

\section{Effect of fertilization of leaf nutrient levels of bearing palms}

The application of $\mathrm{N}$ did not significantly improve leaf $\mathrm{N}$ levels but increased significantly leaf $\mathrm{Ca}$ and $\mathrm{Cl}$ (Table 7). Likewise, $\mathrm{K}$ fertilization did not increase $\mathrm{K}$ contents in the leaf but improved significantly leaf $\mathrm{Cl}$. This trend had been consistent for the last five years of the study.

On the other hand, the application of $\mathrm{Mg}$ especially at higher level did not significantly influence $\mathrm{Mg}$ levels but significantly depressed leaf P. Also, calcium was slightly depressed, indicating the likely existence of $\mathrm{Ca}-\mathrm{Mg}$ and $\mathrm{Mg}-\mathrm{P}$ negative interaction.

The only element that produced consistent response over the years is $\mathrm{Cl}$. Application of which did not only increase concentration of leaf $\mathrm{Cl}$ content but also other elements as leaf $\mathrm{N}, \mathrm{P}$ and $\mathrm{K}$ in some years. This manifests that $\mathrm{Cl}$ is an important element (macronutrient) in coconut as it enhances absorption of other elements except B which appears to be depressed indicating an antagonistic action between these two elements (Cl-B). 
Table 2. Effect of fertilizer treatments on the growth of seedlings in the nursery

\begin{tabular}{|c|c|c|c|c|c|c|c|c|c|c|c|}
\hline \multirow{2}{*}{ Growth Character } & \multicolumn{11}{|c|}{ FERTILIZER TREATMENT } \\
\hline & $\mathrm{N}_{0}$ & $\mathrm{~N}_{1}$ & $\mathrm{~N}_{2}$ & $\mathrm{~K}_{0}$ & $\mathrm{~K}_{1}$ & $\mathrm{~K}_{2}$ & $\mathrm{Mg}_{0}$ & $\mathrm{Mg}_{1}$ & $\mathrm{Mg}_{2}$ & $-\mathrm{Cl}$ & $+\mathrm{Cl}$ \\
\hline \multicolumn{12}{|c|}{ April, 1974 (pre-fertilizer treatment) } \\
\hline Girth (cm) & 6.87 & 6.82 & 6.92 & 6.87 & 6.98 & 6.88 & 6.87 & 6.81 & 6.97 & 6.90 & 6.89 \\
\hline No. of leaflets (leaf 3) & 16.83 & 16.82 & 16.61 & 16.66 & 16.72 & 16.87 & 16.83 & 16.77 & 16.66 & 16.73 & 16.78 \\
\hline No. of leaves & 3.05 & 3.07 & 3.02 & 2.03 & 3.10 & 3.10 & 3.04 & 3.07 & 3.03 & 3.05 & 3.02 \\
\hline \multicolumn{12}{|c|}{ August, 1974 (4 mos. After initial application) } \\
\hline Girth (cm) & 14.64 & 14.64 & 15.27 & 14.43 & 15.22 & 14.92 & 14.54 & 14.73 & 15.32 & 14.59 & $15.12^{\star *}$ \\
\hline No. of leaflets (leaf 3) & 24.96 & 24.50 & 25.08 & 24.52 & 25.47 & 24.52 & 25.56 & 24.48 & 25.49 & 25.26 & 24.43 \\
\hline Height (cm) & 125.84 & 132.59 & 128.25 & 128.84 & 128.30 & 129.54 & 126.67 & 128.30 & 131.71 & 128.69 & 129.09 \\
\hline \multicolumn{12}{|c|}{ November, 1974 (7 mos, after initial application } \\
\hline Girth (cm) & 21.05 & 22.13 & 21.52 & 20.87 & 22.03 & 21.53 & 21.02 & 21.33 & 22.34 & 20.85 & $22.29 *$ \\
\hline No. of leaflets (leaf 3) & 34.49 & 34.24 & 34.06 & 34.21 & 34.79 & 33.80 & 34.19 & 34.21 & 34.41 & 34.28 & 34.25 \\
\hline No. of leaves & 10.99 & 11.12 & 11.11 & 11.14 & 11.13 & 10.95 & 11.04 & 11.14 & 11.04 & 11.09 & 11.06 \\
\hline Height (cm) & 172.40 & 174.50 & 170.26 & 173.33 & 172.69 & 171.09 & 171.97 & 168.87 & 176.28 & 172.04 & 171.70 \\
\hline
\end{tabular}

* Significant

** Highly significant 
Table 3. Effect of fertilizer treatments on the growth of young palms in the field

\begin{tabular}{|c|c|c|c|c|c|c|c|c|c|c|c|}
\hline \multirow{2}{*}{ Growth Character } & \multicolumn{11}{|c|}{ FERTILIZER TREATMENT } \\
\hline & $\mathrm{N}_{0}$ & $\mathrm{~N}_{1}$ & $\mathrm{~N}_{2}$ & $\mathrm{~K}_{0}$ & $\mathrm{~K}_{1}$ & $\mathrm{~K}_{2}$ & $M g_{0}$ & $\mathrm{Mg}_{1}$ & $\mathrm{Mg}_{2}$ & $-\mathrm{Cl}$ & $+\mathrm{Cl}$ \\
\hline \multicolumn{12}{|c|}{ February, 1975 (6 most. From field planting) } \\
\hline Girth (cm) & 18.98 & 18.41 & 18.13 & 17.76 & $19.24^{*}$ & 18.52 & 17.64 & 18.61 & $19.27^{*}$ & 18.10 & $18.91^{\star *}$ \\
\hline No. of leaflets (leaf 3 ) & 44.29 & 44.90 & 45.14 & 44.82 & 45.60 & 43.99 & 43.27 & 45.24 & 45.89 & 44.80 & 44.80 \\
\hline No. of leaves & 11.97 & 12.04 & 11.96 & 11.97 & 12.10 & 11.90 & 11.87 & 12.12 & 11.98 & 12.00 & 11.98 \\
\hline Height (cm) & 165.61 & 168.41 & 165.38 & 166.23 & 167.84 & 165.32 & 163.75 & 168.86 & 166.79 & $168.90^{*}$ & 164.03 \\
\hline \multicolumn{12}{|c|}{ February, 1976 (1.5 yrs. From field-planting) } \\
\hline Girth (cm) & 61.59 & 61.17 & 52.99 & 52.90 & $64.22^{\star *}$ & $62.64^{\star *}$ & 56.42 & 59.30 & $64.04^{*}$ & 56.89 & $62.95^{\star \star}$ \\
\hline No. of leaflets (leaf 3) & 122.25 & 120.15 & 116.44 & 114.83 & $123.05^{\star *}$ & 120.97 ** & 116.10 & 118.71 & $124.04^{\star *}$ & 117.80 & $121.43^{*}$ \\
\hline Accumulated leaves (no.) & 21.40 & 21.39 & 21.01 & 20.87 & $21.59 *$ & 21.34 & 20.91 & 21.39 & 21.51 & 21.16 & 21.38 \\
\hline Height (cm) & 338.83 & 336.21 & $313.53^{*}$ & 302.47 & $347.27^{\star \star}$ & $338.83^{*}$ & 312.49 & 328.31 & $347.77^{\star \star}$ & 326.41 & 332.64 \\
\hline Living fronds (no.) & 8.42 & 8.22 & $7.60^{\star *}$ & 7.63 & $8.31^{* *}$ & $8.31^{* *}$ & 7.88 & 8.09 & 8.27 & 7.68 & $8.84^{* *}$ \\
\hline Leaves produced (no.) (1-1.5 yrs.) & 4.57 & 4.58 & 4.35 & 4.33 & $4.66^{*}$ & 4.53 & 4.38 & 4.51 & 4.62 & 4.43 & $4.58^{*}$ \\
\hline
\end{tabular}

* Significant

** Highly significant 
Count's..... Table 3

\begin{tabular}{|c|c|c|c|c|c|c|c|c|c|c|c|}
\hline \multirow{2}{*}{ Growth Character } & \multicolumn{11}{|c|}{ FERTILIZER TREATMENT } \\
\hline & $\mathrm{N}_{0}$ & $\mathrm{~N}_{1}$ & $\mathrm{~N}_{2}$ & $\mathrm{~K}_{0}$ & $\mathrm{~K}_{1}$ & $\mathrm{~K}_{2}$ & $M g_{0}$ & $M g_{1}$ & $\mathrm{Mg}_{2}$ & $-\mathrm{Cl}$ & $+\mathrm{Cl}$ \\
\hline \multicolumn{12}{|l|}{ August, 1977 (3 years) } \\
\hline Girth $(\mathrm{cm})$ & 127.6 & 130.1 & 124.7 & 120.2 & $131.0^{*}$ & 131.3 & 125.2 & 128.2 & 128.9 & 122.6 & $132.3^{\star *}$ \\
\hline No. of leaflets accumulated & 35.9 & 36.1 & 35.5 & 35.2 & 36.3 & 36.0 & 35.0 & 35.8 & $36.6^{\star}$ & 35.0 & $36.6^{\star *}$ \\
\hline Height (cm) & 623.6 & 639.7 & 613.4 & 587.1 & $641.7^{* \star}$ & $647.9^{* *}$ & 616.4 & 625.5 & 634.8 & 611.6 & $639.6^{* *}$ \\
\hline Living fronds (no.) & 12.9 & 13.1 & 12.4 & 12.0 & $13.3^{*}$ & $13.1^{\star \star}$ & 12.5 & 12.8 & 13.0 & 11.8 & $13.8^{\star *}$ \\
\hline Leaves produced (no.) (2.5-3 yrs). & 5.0 & 5.1 & 4.9 & 4.8 & 5.1 & 5.0 & 4.8 & 5.0 & 5.1 & 4.8 & $5.1^{\star *}$ \\
\hline \multicolumn{12}{|l|}{ August, 1979 (5 yrs.) } \\
\hline Leaves emitted (4.5-5 yrs.) & 7.18 & 7.11 & 7.00 & 7.02 & $7.24^{\star \star}$ & $7.03^{*}$ & 6.87 & $7.11^{\star \star}$ & $7.32^{\star \star}$ & 6.93 & $7.27^{\star \star}$ \\
\hline Living fronds (no.) & 21.58 & 22.53 & 22.09 & 21.54 & 22.59 & 22.08 & 21.33 & 22.23 & 22.64 & 20.60 & $23.54^{\star *}$ \\
\hline Accumulated leaves (no.) & 61.97 & 62.31 & 61.42 & 60.64 & $62.93^{\star *}$ & $62.12^{\star *}$ & 60.84 & 62.0 & $63.35^{\star *}$ & 60.37 & $64.43^{* *}$ \\
\hline Trunk height (cm) & 91.43 & 101.13 & 93.31 & 79.26 & $103.73^{\star *}$ & $102.88^{\star \star}$ & 86.19 & $97.47^{\star \star}$ & 102.21 & 87.34 & $103.24^{\star *}$ \\
\hline
\end{tabular}

* Significant

** Highly significant 
Table 4. Main effect of $\mathrm{N}, \mathrm{K}, \mathrm{Mg}$, and $\mathrm{Cl}$ on the annual nut production for the last 5 years

\begin{tabular}{|c|c|c|c|c|c|}
\hline TREATMENT & 1984 & 1985 & 1986 & 1987 & 1988 \\
\hline \multicolumn{6}{|c|}{ N U M B E R } \\
\hline N0 & 26.2 & 71.4 & 42.6 & 70.2 & 55.7 \\
\hline N1 & 33.3 & 72.5 & 44.8 & 67.6 & 60.2 \\
\hline N2 & 34.0 & $79.8^{*}$ & 44.0 & 75.6 & 60.9 \\
\hline K0 & 30.3 & 68.4 & 40.8 & 65.0 & 56.4 \\
\hline K1 & 30.4 & $77.0^{*}$ & 43.9 & $76.8^{*}$ & 59.4 \\
\hline$\overline{\mathrm{Mg} 0}$ & 34.3 & $\overline{73.8}$ & 44.6 & 70.0 & 61.5 \\
\hline Mg1 & 29.3 & 72.4 & 42.8 & 72.0 & 56.6 \\
\hline $\mathrm{Mg} 2$ & 29.9 & 77.4 & 43.9 & 71.4 & 59.8 \\
\hline LSD 0.05 & 9.64 & 8.067 & 9.616 & 7.162 & 8.828 \\
\hline 0.01 & 13.33 & 11.156 & 13.298 & 9.905 & 12.209 \\
\hline$-\mathrm{Cl}$ & 26.0 & 63.4 & 40.0 & 63.1 & 50.8 \\
\hline$+\mathrm{Cl}$ & $36.3 * *$ & $85.7 * *$ & $47.6^{*}$ & $79.2 * *$ & $67.1 * *$ \\
\hline LSD 0.05 & 5.51 & 5.857 & 7.450 & 5.947 & 6.234 \\
\hline 0.01 & 7.56 & 8.045 & 10.232 & 8.168 & 8.562 \\
\hline
\end{tabular}

* Significant

** Highly significant

Table 5. Main effect of $\mathrm{N}, \mathrm{K}, \mathrm{Mg}, \& \mathrm{Cl}$ on yearly average copra weight per nut for the last five years

\begin{tabular}{|c|c|c|c|c|c|}
\hline TREATMENT & 1984 & 1985 & 1986 & 1987 & 1988 \\
\hline \multicolumn{6}{|c|}{ G R A M } \\
\hline N0 & 275.5 & 237.1 & 280.3 & 237.0 & 237.1 \\
\hline N1 & 283.5 & $258.5^{*}$ & 294.6 & 245.5 & $253.8 * *$ \\
\hline $\mathrm{N} 2$ & 297.5 & $267.3^{*}$ & $311.3^{*}$ & $262.4^{*}$ & $261.4^{* *}$ \\
\hline K0 & 268.9 & 246.0 & 279.7 & 235.7 & 238.7 \\
\hline K1 & 291.7 & 251.7 & $303.8 *$ & 256.0 & $254.7 * *$ \\
\hline $\mathrm{K} 2$ & $294.2 *$ & 265.2 & $302.6^{*}$ & 253.2 & $259.9 * *$ \\
\hline Mg0 & 279.4 & 245.3 & 282.4 & 235.3 & 244.0 \\
\hline Mg1 & 282.9 & 256.6 & 298.3 & 255.5 & 254.0 \\
\hline $\mathrm{Mg} 2$ & 293.5 & 261.0 & 305.4 & 254.0 & 254.4 \\
\hline LSD 0.05 & 22.78 & 17.73 & 17.9 & 20.5 & 11.1 \\
\hline 0.01 & 31.51 & 24.52 & 24.8 & 28.4 & 15.3 \\
\hline$-\mathrm{Cl}$ & 250.4 & 222.8 & 254.7 & 213.4 & 219.8 \\
\hline$+\mathrm{Cl}$ & $319.4 * *$ & $285.8 * *$ & $336.0 * *$ & $283.2 * *$ & $281.8 * *$ \\
\hline LSD 0.05 & 16.28 & 13.08 & 18.52 & 14.30 & 14.43 \\
\hline 0.01 & 22.56 & 17.96 & 25.43 & 19.64 & 19.82 \\
\hline
\end{tabular}


Table 6. Main effect of $\mathrm{N}$. K. Mg \& $\mathrm{Cl}$ on the annual copra production per tree for the last five years

\begin{tabular}{|c|c|c|c|c|c|}
\hline TREATMENT & 1984 & 1985 & 1986 & 1987 & 1988 \\
\hline \multicolumn{6}{|c|}{ K I LO G R A M } \\
\hline N0 & 7.5 & 17.9 & 12.4 & 17.2 & 13.5 \\
\hline N1 & 9.6 & 19.4 & 13.4 & 17.0 & $15.6^{*}$ \\
\hline $\mathrm{N} 2$ & 10.1 & $21.7 * *$ & 13.5 & 20.1 ** & $16.2 *$ \\
\hline K0 & 8.2 & 17.4 & 11.6 & 15.8 & 13.7 \\
\hline K1 & 9.0 & $20.3^{* *}$ & 13.3 & $20.1 * *$ & 15.4 \\
\hline $\mathrm{K} 2$ & 9.9 & $21.3 * *$ & 14.4 & $18.5^{*}$ & $16.2 *$ \\
\hline$\overline{\mathrm{Mg} 0}$ & 9.8 & 18.8 & 12.9 & 17.1 & $\overline{15.6}$ \\
\hline Mg1 & 8.5 & 19.6 & 13.1 & 18.8 & 14.7 \\
\hline Mg2 & 8.8 & 20.6 & 13.2 & 18.5 & 15.1 \\
\hline LSD 0.05 & 2.613 & 2.370 & 3.001 & 2.001 & 2.076 \\
\hline 0.01 & 3.613 & 3.278 & 4.150 & 2.767 & 2.870 \\
\hline$-\mathrm{Cl}$ & 6.5 & 14.3 & 10.3 & 13.5 & 11.2 \\
\hline$+\mathrm{Cl}$ & $11.6^{* *}$ & $25.1 * *$ & $15.9 * *$ & $22.7 * *$ & $19.1 * *$ \\
\hline LSD 0.05 & 1.706 & 1.863 & 2.575 & 1.826 & 1.918 \\
\hline 0.01 & 2.343 & 2.558 & 3.536 & 2.508 & 2.634 \\
\hline
\end{tabular}

* Significant

** Highly significant 
Table 7. Effect of fertilizer treatments on leaf nutrient of bearing palms

\begin{tabular}{|c|c|c|c|c|c|c|c|c|c|c|c|}
\hline \multirow{2}{*}{$\begin{array}{c}\text { Sampling } \\
\text { Year/Leaf } \\
\text { Nutrient (\%) }\end{array}$} & \multicolumn{11}{|c|}{ FERTILIZER TREATMENT } \\
\hline & $\mathrm{N}_{0}$ & $\mathrm{~N}_{1}$ & $\mathrm{~N}_{2}$ & $\mathrm{~K}_{0}$ & $\mathrm{~K}_{1}$ & $\mathrm{~K}_{2}$ & $\mathrm{Mg}_{0}$ & $\mathrm{Mg}_{1}$ & $\mathrm{Mg}_{2}$ & $-\mathrm{Cl}$ & $+\mathrm{Cl}$ \\
\hline \multicolumn{12}{|l|}{1984 (leaf 14) } \\
\hline $\mathrm{N}$ & 1.795 & 1.814 & 1.827 & 1.787 & 1.834 & 1.815 & 1.820 & 1.809 & 1.807 & 1.783 & $1.841^{\text {** }}$ \\
\hline$P$ & 0.150 & 0.151 & 0.155 & 0.151 & 0.153 & 0.153 & 0.153 & 0.155 & 0.149 & 0.152 & 0.152 \\
\hline $\mathrm{K}$ & 1.226 & 1.218 & 1.274 & 1.209 & 1.252 & 1.257 & 1.174 & $1.255^{\star}$ & $1.288^{*}$ & 1.210 & $1.269 *$ \\
\hline $\mathrm{Ca}$ & 0.443 & 0.471 & $0.482^{*}$ & 0.451 & 0.472 & 0.472 & 0.479 & 0.468 & 0.449 & 0.460 & 0.470 \\
\hline $\mathrm{Mg}$ & 0.218 & 0.219 & 0.228 & 0.224 & 0.221 & 0.220 & 0.216 & 0.225 & 0.224 & 0.218 & 0.226 \\
\hline $\mathrm{Na}$ & 0.051 & 0.046 & 0.055 & 0.056 & 0.051 & 0.045 & 0.048 & 0.053 & 0.050 & 0.050 & 0.051 \\
\hline $\mathrm{Cl}$ & 0.212 & $0.294^{\star \star}$ & $0.400 * *$ & 0.239 & $0.314^{\star \star}$ & $0.314^{\star *}$ & $0.352^{\star *}$ & 0.311 & 0.318 & 0.085 & $0.518 *$ \\
\hline B (ppm) & 10.9 & 10.9 & 10.5 & 10.8 & 10.8 & 10.7 & 10.6 & 10.7 & 11.0 & 11.4 & 10.1 \\
\hline \multicolumn{12}{|l|}{1985 (leaf 14) } \\
\hline $\mathrm{N}$ & 1.822 & 1.852 & 1.867 & 1.807 & 1.864 & 1.870 & $1.893^{*}$ & 1.844 & 1.804 & 1.793 & $1.901 *$ \\
\hline$P$ & 0.139 & 0.138 & 0.141 & 0.137 & 0.140 & 0.140 & 0.143 & 0.140 & $0.135^{\star *}$ & 0.138 & 0.140 \\
\hline $\mathrm{K}$ & 1.556 & 1.544 & 1.532 & 1.535 & 1.567 & 1.530 & 1.546 & 1.538 & 1.548 & 1.540 & 1.548 \\
\hline $\mathrm{Ca}$ & 0.327 & 0.342 & 0.348 & 0.336 & 0.335 & 0.345 & 0.335 & 0.336 & $0.325^{\star}$ & 0.340 & 0.337 \\
\hline $\mathrm{Mg}$ & 0.180 & 0.179 & 0.178 & 0.180 & 0.176 & 0.181 & 0.179 & 0.179 & 0.179 & 0.182 & 0.176 \\
\hline $\mathrm{Na}$ & 0.177 & 0.227 & $0.327^{\star \star}$ & 0.173 & $0.264^{\star *}$ & 0.294 & 0.265 & 0.243 & 0.223 & 0.068 & $0.419 * *$ \\
\hline $\mathrm{Cl}$ & 0.612 & $0.166^{*}$ & $0.170 * \star$ & 0.160 & $0.169 * *$ & $0.169 * *$ & 0.166 & 0.167 & 0.165 & 0.165 & 0.167 \\
\hline B (ppm) & 9.2 & 9.4 & 8.9 & 9.1 & 9.2 & 9.1 & 9.1 & 9.1 & 9.3 & 9.5 & 8.8 \\
\hline \multicolumn{12}{|l|}{1986 (leaf 14) } \\
\hline $\mathrm{N}$ & 1.996 & 2.004 & 1.003 & 1.993 & 2.012 & 1.998 & 2.106 & 1.998 & 1.990 & 1.966 & $2.036^{\star *}$ \\
\hline$P$ & 0.145 & 0.142 & 0.145 & 0.144 & 0.143 & 0.143 & 0.147 & 0.143 & $0.140^{*}$ & 0.142 & 0.145 \\
\hline $\mathrm{K}$ & 1.381 & 1.360 & 1.354 & 1.352 & 1.372 & 1.372 & 1.363 & 1.379 & 1.354 & 1.355 & 1.378 \\
\hline $\mathrm{Ca}$ & 0.386 & $0.413^{*}$ & $0.428^{*}$ & 0.397 & 0.411 & 0.419 & 0.420 & 0.405 & 0.403 & 0.403 & 0.415 \\
\hline
\end{tabular}




\begin{tabular}{|c|c|c|c|c|c|c|c|c|c|c|c|}
\hline $\mathrm{Mg}$ & 0.182 & 0.191 & 0.192 & 0.187 & 0.182 & 0.195 & 0.194 & 0.183 & 0.188 & 0.189 & 0.187 \\
\hline $\mathrm{Na}$ & 0.052 & 0.051 & 0.053 & 0.055 & 0.046 & 0.056 & 0.051 & 0.052 & 0.054 & 0.053 & 0.052 \\
\hline $\mathrm{Cl}$ & 0.215 & $0.299 * \star$ & $0.373^{\star \star}$ & 0.212 & $0.319^{* *}$ & $0.355^{\star \star}$ & 0.289 & 0.298 & 0.300 & 0.072 & 0.519 \\
\hline$S$ & 0.181 & 0.183 & 0.186 & 0.183 & 0.181 & 0.184 & 0.183 & 0.185 & 0.181 & 0.184 & 0.182 \\
\hline $\mathrm{B}(\mathrm{ppm})$ & 11.2 & 10.6 & 10.6 & 10.8 & 10.9 & 10.7 & 10.9 & 10.5 & 11.0 & 11.3 & $10.2^{\star \star}$ \\
\hline \multicolumn{12}{|c|}{1987 (leaf 14) } \\
\hline $\mathrm{N}$ & 1.875 & 1.904 & 1.892 & 1.870 & 1.900 & 1.891 & 1.914 & 1.875 & 1.883 & 1.848 & $1.933^{\star \star}$ \\
\hline$P$ & 0.153 & 0.152 & 0.151 & 0.151 & 0.151 & 0.154 & 0.155 & 0.153 & $0.148^{*}$ & 0.151 & 0.153 \\
\hline K & 1.633 & 1.659 & 1.613 & 1.612 & 1.654 & 1.639 & 1.637 & 1.627 & 1.641 & 1.596 & $1.694^{\star \star}$ \\
\hline $\mathrm{Ca}$ & 0.327 & 0.351 & 0.343 & 0.328 & 0.335 & 0.357 & 0.345 & 0.343 & 0.332 & 0.344 & 0.336 \\
\hline $\mathrm{Mg}$ & 0.216 & 0.190 & 0.191 & 0.187 & 0.213 & 0.196 & 0.190 & 0.220 & 0.186 & 0.190 & 0.207 \\
\hline $\mathrm{Na}$ & 0.074 & 0.063 & 0.069 & 0.070 & 0.071 & 0.064 & 0.068 & 0.070 & 0.067 & 0.067 & 0.070 \\
\hline $\mathrm{Cl}$ & 0.281 & 0.325 & 0.332 & 0.223 & $0.331^{\star *}$ & $0.386^{\star \star}$ & 0.270 & 0.317 & 0.352 & 0.102 & $0.532^{\star *}$ \\
\hline$S$ & 0.148 & 0.147 & 0.154 & 0.147 & 0.151 & 0.151 & 0.152 & 0.148 & 0.149 & 0.150 & 0.150 \\
\hline B (ppm) & 9.5 & 9.4 & 9.0 & 9.2 & 9.3 & 9.4 & 9.4 & 9.4 & 9.2 & 9.5 & $9.1^{\star \star}$ \\
\hline \multicolumn{12}{|c|}{1988 (leaf 14) } \\
\hline N & 1.987 & 2.036 & 2.012 & 2.012 & 2.023 & 2.015 & 2.020 & 2.009 & 2.007 & 1.959 & $2.064^{\star \star}$ \\
\hline$P$ & 0.157 & 0.159 & 0.158 & 0.156 & 0.159 & 0.159 & 0.162 & 0.158 & $0.154^{*}$ & 0.155 & $0.166^{\star \star}$ \\
\hline K & 1.609 & 1.569 & 1.566 & 1.547 & 1.596 & 1.601 & 1.571 & 1.590 & 1.583 & 1.595 & 1.568 \\
\hline $\mathrm{Ca}$ & 0.359 & $0.381^{*}$ & $0.385^{\star}$ & 0.373 & 0.368 & 0.384 & 0.388 & 0.374 & 0.363 & 0.370 & 0.380 \\
\hline $\mathrm{Mg}$ & 0.215 & 0.216 & 0.227 & 0.225 & 0.215 & 0.218 & 0.218 & 0.216 & 0.224 & 0.219 & 0.219 \\
\hline $\mathrm{Na}$ & 0.043 & 0.045 & 0.048 & 0.049 & 0.045 & 0.042 & 0.045 & 0.047 & 0.044 & 0.046 & 0.045 \\
\hline $\mathrm{Cl}$ & 0.211 & $0.296^{* *}$ & $0.361^{\star *}$ & 0.212 & $0.312^{\star *}$ & 0.343 & 0.290 & 0.285 & 0.292 & 0.081 & 0.497 \\
\hline$S$ & 0.169 & 0.171 & 0.174 & 0.169 & 0.172 & 0.172 & 0.170 & 0.171 & 0.172 & 0.169 & 0.172 \\
\hline B (ppm) & 8.8 & 8.5 & 8.4 & 8.6 & 8.6 & 8.5 & 8.1 & 8.1 & 8.8 & 9.0 & 8.2 \\
\hline
\end{tabular}


Table 8. Correlation between coconut yield and leaf nutrient levels (1984)

\begin{tabular}{|c|c|c|c|c|c|c|c|c|c|c|c|c|}
\hline & $\begin{array}{l}\text { NUTI } \\
\text { PALM }\end{array}$ & $\begin{array}{c}\text { COPRA/ } \\
\text { NUT }\end{array}$ & $\begin{array}{l}\text { COPRA/ } \\
\text { PALM }\end{array}$ & N & $P$ & K & $\mathrm{Ca}$ & $\mathrm{Mg}$ & $\mathrm{Na}$ & $\mathrm{Cl}$ & $S$ & B \\
\hline NUT/TREE & 1.000 & & & & & & & & & & & \\
\hline COPRA/NUT & 0.150 & 1.000 & & & & & & & & & & \\
\hline COPRA/TREE & $0.938^{\star *}$ & $0.423^{\star \star}$ & 1.000 & & & & & & & & & \\
\hline N & 0.234 & $0.325^{\star}$ & $0.300^{*}$ & 1.000 & & & & & & & & \\
\hline $\mathrm{P}$ & 0.084 & 0.094 & 0.139 & 0.218 & 1.000 & & & & & & & \\
\hline K & 1.109 & 0.144 & 0.193 & -0.088 & 0.179 & 1.000 & & & & & & \\
\hline $\mathrm{Ca}$ & $0.340^{*}$ & 0.192 & $-0.378^{* *}$ & 0.206 & $0.540^{\star *}$ & -0.046 & 1.000 & & & & & \\
\hline $\mathrm{Mg}$ & -0.299 & $0.336^{*}$ & -0.100 & 0.170 & $0.411^{* *}$ & 0.092 & 0.222 & 1.000 & & & & \\
\hline $\mathrm{Na}$ & $-0.0421^{* *}$ & 0.110 & -0.318 & 0.232 & 0.065 & 0.090 & -0.229 & $0.413^{*}$ & 1.000 & & & \\
\hline $\mathrm{Cl}$ & $0.419 * *$ & $0.692^{\star \star}$ & 0.648 ** & $0.309^{*}$ & 0.125 & $0.405^{\star \star}$ & $0.302^{*}$ & $0.233^{\star \star}$ & 0.034 & 1.000 & & \\
\hline $\mathrm{S}$ & 0.139 & 0.035 & 0.110 & $0.487^{* *}$ & 0.294 & -0.059 & 0.286 & -0.009 & -0.270 & 0.050 & 1.000 & \\
\hline B & $-0.451^{\star *}$ & $-0.417^{\star \star}$ & -0.584 & -0.124 & -0.218 & $-0.311^{*}$ & -0.174 & -0.254 & -0.176 & $-0.672^{*}$ & 0.030 & 1.000 \\
\hline
\end{tabular}

Tabular $\mathrm{r}$ - values

$0.05=0.268$

$0.01=0.353$ 
Table 9. Correlation between cpcpmit yield and left nutrient levels (1986)

\begin{tabular}{|c|c|c|c|c|c|c|c|c|c|c|c|c|}
\hline & $\begin{array}{l}\text { NUT/ } \\
\text { PALM }\end{array}$ & $\begin{array}{c}\text { COPRA/ } \\
\text { NUT }\end{array}$ & $\begin{array}{l}\text { COPRAl } \\
\text { PALM }\end{array}$ & $\mathrm{N}$ & $P$ & K & $\mathrm{Ca}$ & $\mathrm{Mg}$ & $\mathrm{Na}$ & $\mathrm{Cl}$ & $\mathrm{S}$ & B \\
\hline NUT/TREE & 1.000 & & & & & & & & & & & \\
\hline COPRA/NUT & $0.386^{\star *}$ & 1.000 & & & & & & & & & & \\
\hline COPRA/TREE & $0.867^{\star *}$ & $0.664^{\star *}$ & 1.000 & & & & & & & & & \\
\hline N & 0.260 & $0.473^{\star \star}$ & $0.495^{\star \star}$ & 1.000 & & & & & & & & \\
\hline$P$ & -0.100 & 0.150 & 0.045 & 0.429 & 1.000 & & & & & & & \\
\hline K & -0.073 & -0.81 & -0.023 & 0.049 & 0.128 & 1.000 & & & & & & \\
\hline $\mathrm{Ca}$ & 0.242 & $0.277^{\star}$ & $0.334^{*}$ & 0.223 & 0.144 & -0.240 & 1.000 & & & & & \\
\hline $\mathrm{Mg}$ & -0.023 & 0.135 & -0.047 & -0.186 & 0.033 & $0.397^{\star *}$ & 0.158 & 1.000 & & & & \\
\hline $\mathrm{Na}$ & 0.025 & 0.106 & -0.008 & 0.041 & 0.125 & -0.100 & -0.285 & 0.174 & 1.000 & & & \\
\hline $\mathrm{Cl}$ & $0.288^{*}$ & $0.844^{\star *}$ & $0.617^{\star \star}$ & $0.492^{\star *}$ & 0.232 & 0.009 & $0.396^{* *}$ & 0.083 & 0.035 & 1.000 & & \\
\hline$S$ & 0.082 & -0.010 & 0.038 & $0.307^{\star}$ & 0.234 & -0.021 & 0.029 & 0.068 & 0.059 & -0.057 & 1.000 & \\
\hline B & -0.178 & $-0.533^{\star \star}$ & $-0.337^{\star *}$ & -0.136 & -0.285 & -0.019 & 0.043 & -0.222 & 0.24 & $0.527^{\star \star}$ & -0.038 & 1.000 \\
\hline
\end{tabular}

Tabular $\mathrm{r}$ - values

$0.05=0.268$

$0.01=0.353$ 
Table 10. Correlation between coconut yield and leaf nutrient levels 91988)

\begin{tabular}{|c|c|c|c|c|c|c|c|c|c|c|c|c|}
\hline & $\begin{array}{l}\text { NUT/ } \\
\text { PALM }\end{array}$ & $\begin{array}{c}\text { COPRAI } \\
\text { NUT }\end{array}$ & $\begin{array}{c}\text { COPRA } \\
\text { PALM }\end{array}$ & $\mathrm{N}$ & $P$ & $\mathrm{~K}$ & $\mathrm{Ca}$ & $\mathrm{Mg}$ & $\mathrm{Na}$ & $\mathrm{Cl}$ & $S$ & $B$ \\
\hline NUT/TREE & 1.000 & & & & & & & & & & & \\
\hline COPRA/NUT & $0.442^{* *}$ & 1.000 & & & & & & & & & & \\
\hline COPRA/TREE & $0.850^{* *}$ & 0.768 & 1.000 & & & & & & & & & \\
\hline $\mathrm{N}$ & $0.524^{\star *}$ & $0.605^{\star *}$ & 0.768 & 1.000 & & & & & & & & \\
\hline$P$ & -0.127 & -0.167 & $0.605^{\star *}$ & -0.042 & 1.000 & & & & & & & \\
\hline K & 0.168 & -0.114 & -0.167 & -0.056 & -0.080 & 1.000 & & & & & & \\
\hline $\mathrm{Ca}$ & $0.372^{* *}$ & 0.095 & 0.114 & 0.150 & -0.045 & -0.209 & 1.000 & & & & & \\
\hline $\mathrm{Mg}$ & -0.446 & 0.073 & 0.095 & -0.180 & 0.004 & -0.552 & 0.097 & 1.000 & & & & \\
\hline $\mathrm{Na}$ & -0.044 & 0.069 & 0.073 & 0.190 & -0.130 & -0.238 & 0.187 & 0.229 & 1.000 & & & \\
\hline $\mathrm{Cl}$ & $0.536^{\star *}$ & $0.885^{\star *}$ & 0.069 & $0.651^{\star *}$ & -0.163 & -0.178 & 0.266 & 0.078 & 0.153 & 1.000 & & \\
\hline$S$ & $0.329 *$ & $0.436^{\star *}$ & $0.885^{\star *}$ & $0.459 * *$ & 0.187 & -0.049 & 0.080 & -0.004 & 0.074 & 0.437 ** & 1.000 & \\
\hline B & -0.147 & $-0460 * *$ & $0.460^{* *}$ & $-0.393^{* *}$ & -0.108 & -0.018 & -0.089 & -0.071 & -0.236 & $-0.492^{\star \star}$ & -0.238 & 1.000 \\
\hline
\end{tabular}

Tabular $\mathrm{r}-$ values

$0.05=0.268 \quad 0.01=0.353$ 
Table 11. Suggested guide on Cl needs (nursery to full bearing) of coconuts, local tall variety

\begin{tabular}{|l|r|r|r|r|}
\hline \multirow{2}{*}{ STAGE AND AGE } & \multirow{2}{*}{$\begin{array}{c}\text { Cl/PALM } \\
(\mathrm{g})\end{array}$} & \multicolumn{2}{|c|}{ FERTILIZER SOURCE* $(\mathrm{g})$} \\
\cline { 3 - 3 } & & $\mathrm{KCl}$ & $\mathrm{NaCl}$ \\
\hline Nursery: & 10 & 25 & & 20 \\
2 mos. & 20 & 50 & & 40 \\
5 mos. & & & & \\
\hline Field: & 40 & 100 & & 80 \\
Field-planting & 100 & 230 & & 400 \\
6 mos. & 200 & 450 & & 800 \\
1 yr & 400 & 900 & & 1,200 \\
2 yrs & 600 & 1,400 & & 1,600 \\
3 yrs & 800 & 1,800 & & 2,000 \\
4 yrs & 1,000 & 2,250 & & \\
5 yrs \& above & & & & \\
\hline
\end{tabular}

$* \mathrm{KCl}=44 \% \quad \mathrm{Cl}, 50 \% \mathrm{~K} \quad \mathrm{NaCl}=50 \% \mathrm{Cl}$

\section{Correlation and analysis}

The correlation analyses (Tables $8,9,10$ ) show that in general both leaf $\mathrm{N}$ and CI were positively and significantly related with nut per palm, copra per nut and copra per palm with leaf-Cl effect very consistent over the years. Calcium was correlated with nut and copra yield but the relationship was inconsistent over the years. Sulfur is also correlated to nut and copra yield but was only observed in the last year of the study. On the other hand leaf-Mg was negatively correlated with yield which is surprising because aside from the low levels of $\mathrm{Mg}$ in the leaves and although no main effects of $\mathrm{Mg}$ was observed, it interacted with $\mathrm{N}$ in improving copra yield per nut. Boron is another element which was negatively related with yield which could be due also to its antagonistic reaction with $\mathrm{Cl}$.

A stepwise regression analysis made for the year 1988 indicated that $\mathrm{Cl}$ is the main determinant for each of the three production parameters nut/palm, copra/nut and copra/palm, accounting for 29,78 and $68 \%$ contribution, respectively. These results suggest that the yield improvement observed in this study was primarily due to the correction of $\mathrm{Cl}$ deficiency. This generally improved the physiology and nutrient utilization efficiency (conversion to economic yield as nuts and copra) of the palms. Several workers reported positive correlation of leaf CI and copra yield (Ollagnier and Ochs, 1971; Vexkull, 1972; prudente and Mendoza, 1976).

\section{Suggested nutritional Needs}

Based on the results of this study, a suggested guide on the nutritional needs of local tall palms for $\mathrm{Cl}$ from nursery to full bearing is presented in Table 11. During the nursery stage, a total of $30 \mathrm{~g} \mathrm{Cl}$ per seedling; while in the field, the requirement increases with age of coconut ( $40 \mathrm{~g}$ to 1,000 g per palm), leveling off at 5 years from field planting. Thus at bearing stage and onwards $1 \mathrm{~kg} \mathrm{Cl}$ per tree is the estimated $\mathrm{Cl}$ need of the coconut for normal development and production. In normal years, under Davao conditions the average yields of coconut was 70 nuts per tree/year ( $20.7 \mathrm{~kg}$ copra per 
tree/year) with adequate $\mathrm{Cl}$ nutrition as in this study. So, at the average plant population of 110 trees/ha this means an annual yield of 77,00 nuts or 2.3 tons copra per ha. The information presented in this report may serve as a useful reference in the fertilization of coconuts, particularly in inland areas.

\section{SUMMARY AND CONCLUSION}

It is indispensable to know the critical levels of nutrients as they indicate the nutritional status of the palms, which serves as the basis of diagnosing the fertilizer needs of coconuts under different agro climatic conditions.

In the nursery and in the field, $\mathrm{Cl}$ application showed a consistent positive influence on the stem girth up to 4 years; leaflets up to 2 years; living fronds and number of leaves emitted every 6 months up to 5 years. One year from field-planting and 5 years thereafter, consistent significant response of the palms on the basis of almost ail growth characters was observed with the addition of $\mathrm{K}$ and $\mathrm{Cl}$ indicating the added advantage of $\mathrm{KCl}$ as source of $\mathrm{K}$ over $\mathrm{KISO}_{4}-$ Moreover, $\mathrm{Mg}$ addition to young palms appears to be beneficial for accelerated development. At 5 years from field-planting, $\mathrm{Cl}$-applied palms had $115 \%$ more flowering coconuts than minus $\mathrm{Cl}$ palms. During the bearing stage (6th - 14th year) $\mathrm{Cl}$ application consistently increased nut production, copra weight per nut and copra yield per palm which were positively correlated with leaf $\mathrm{Cl}$. The average copra weight per nut for palms applied with $\mathrm{Cl}(1 \mathrm{~kg} \mathrm{Cl} /$ palm/year) was $320 \mathrm{~g}$, compared to $223 \mathrm{~g}$ per nut for minus $\mathrm{Cl}$ palms. The positive influence observed on $\mathrm{N}, \mathrm{K}$ and $\mathrm{Mg}$ on yield was inconsistent over the years. Likewise, interaction effects of nutrient on yield and on leaf nutrient levels were inconsistent, indicating the adequacy of these nutrients in the soil during some years.

As suggested guide on the nutritional needs of the local tall palms for $\mathrm{Cl}$ from the nursery to full-bearing is presented (Table 11). 


\section{REFERENCES}

MAGAT, S. S., V. L CADIGAL and J. A. HABANA. 1975. Yield improvement of coconuts in elevated inland area of Davao (Phil.) by KCI fertilization. Oleagineux 30 (10) 413-416.

and R. L. PRUDENTE. 1975. Progress of mineral nutrition studies on coconut in the Philippines. Fourth Session of the FAO Technical Working Party on Coconut Production and Processing. Kingston, Jamaica, Sept. 1424, 1975.

R. Z. MARGATE AND R. L. PRUDENTE. 1977. Utilization of common table salt as a fertilizer and for the control of leaf spot disease of coconut seedlings. Phil. J. Coconut Studies. 2 (3): 39-45.

and L. G. OGUIS. 1979. Early results of a study on the chlorine nutritional needs of coconut in the Philippines. Paper presented during the Fifth Session of the FAO Tech. Working Party on Coconut Production, Protection and Processing, Dec. 3-8, 1979, Manila, Philippines.

MARAVILLA, J. N., R. L. PRUDENTE and S. S. MAGAT. 1978. Fertilizer requirement of coconut seedlings grown on three major coconut soils of Davao. Proc. $9^{\text {th }}$ Ann. Scientific Meeting, CSSP. May 2-5, 1978, Iloilo City.

MARGATE, R. Z., S. S. MAGAT, L. M. ALFORJA and J. A. HABANA. 1978. A long term, KCI fertilization of bearing coconuts in an inland-upland area of Davao. Phil. J. Coconut Studies 3 (4): $1-4$.

MENDOZA, A. M. R. and R. L. PRUDENTE. 1972. An influence of NPK fertilizers on coconut. Proc. CSSP, Cagavan de Oro City, May 1972.

OGUIS, L. G., S. S. MAGAT and R. Z. MARGATE. 1979. The effect of chlorine sources and ammonium sulfate on the growth of coconut seedlings. Phil. J. Coconut Studies. 14 (3): 25-38.

PRUDENTE, R. L. and A. M. R. MENDOZA. 1976. Response of inland coconuts to inorganic fertilization from field-planting. Phil. J. Coconut Studies. 1 (1): 27-36. 
Appendix

Table. 1 Soil analysis of Tugbok soil, PCA-Davao Research Center, Bago-Oshiro, Davao City*

\begin{tabular}{|c|c|c|}
\hline PROPERTY & $\begin{array}{c}\text { SURFACE SOIL } \\
(0-17 \mathrm{~cm})\end{array}$ & $\begin{array}{c}\text { SUB-SOILS } \\
(18-80 \mathrm{~cm})\end{array}$ \\
\hline \multicolumn{3}{|l|}{ Chemical } \\
\hline PH (1:1/soil: $\left.\mathrm{H}_{2} \mathrm{O}\right)$ & 6.50 & 6.70 \\
\hline Organic Mater (\%) & 1.73 & 1.40 \\
\hline Available $\mathrm{p}(\mathrm{ppm})$ & 19.00 & 11.00 \\
\hline $\mathrm{K}-\mathrm{H}_{2} \mathrm{SO}_{4}$ extractable (ppm) & 624.00 & 677.00 \\
\hline \multicolumn{3}{|l|}{ Exchangeable bases (m.e./100 g): } \\
\hline $\mathrm{Ca}$ & 11.40 & 12.30 \\
\hline $\mathrm{Mg}$ & 5.30 & 6.40 \\
\hline $\mathrm{K}$ & 0.45 & 0.29 \\
\hline $\mathrm{Na}$ & 0.08 & 0.18 \\
\hline CEC (M.E./100 g) & 25.20 & 26.70 \\
\hline Base Saturation (\%) & 68.00 & 72.00 \\
\hline \multicolumn{3}{|l|}{ Physical } \\
\hline Texture & Clay loam & Clay \\
\hline$\%$ Sand & 32.9 & 23.3 \\
\hline$\%$ Silt & 33.3 & 28.9 \\
\hline$\%$ Clay & 33.9 & 47.8 \\
\hline Bulk density (g/cc) & 1.6 & 1.5 \\
\hline Total porosity $(\%)$ & 40.6 & 45.3 \\
\hline \multicolumn{3}{|l|}{ Soil moisture (\%): } \\
\hline Field capacity & 32.6 & 40.60 \\
\hline Permanent wilting point & 17.6 & 26.7 \\
\hline Available moisture & 14.9 & 13.8 \\
\hline
\end{tabular}

*Analyzed at the Bureau of Soils Laboratory (Manila). 